\title{
Externalizing the Core Principles of the Departmental Action Team (DAT) model
}

\author{
Joel C. Corbo, ${ }^{1}$ Gina M. Quan, ${ }^{1, *}$ Karen Falkenberg, ${ }^{2}$ Christopher Geanious, ${ }^{2}$ \\ Courtney Ngai, ${ }^{2}$ Mary E. Pilgrim, ${ }^{3}$ Daniel L. Reinholz, ${ }^{3}$ and Sarah Wise ${ }^{4}$ \\ ${ }^{1}$ Center for STEM Learning, University of Colorado, Boulder, CO \\ ${ }^{2}$ The Institute for Learning and Teaching, Colorado State University, Fort Collins, CO \\ ${ }^{3}$ Department of Mathematics, San Diego State University, San Diego, CA \\ ${ }^{4}$ Academic Technology Design Team, University of Colorado Boulder, Boulder, CO
}

\begin{abstract}
Departmental Action Teams (DATs) are departmentally-based working groups of faculty, students, and staff aimed at achieving sustained departmental change related to undergraduate education. DATs have been conceptualized and are facilitated by members of our project team based on a set of Core Principles. These principles serve both as guides in the design of DATs and targets for the kinds of culture we aspire to create through our facilitation. In this paper, we describe our Core Principles, including theoretical underpinnings and a brief implementation example for each. We argue that articulating principles is a critical component of externalizing a complex change effort and that our Core Principles are applicable beyond the DAT model.
\end{abstract}

\section{INTRODUCTION}

Prior research on educational innovations suggests that changes to undergraduate education that focus on individual instructors or courses are difficult to sustain [1]. This difficulty arises largely because cultural features of the broader department (e.g., norms, values, reward structures) can significantly impact the adoption of change efforts, including by hindering them $[2,3]$. This motivates a focus on change efforts at the department scale, rather than on courses or instructors.

We cultivate department-level changes through Departmental Action Teams (DATs), a new type of departmentallybased working group that aims to sustainably improve undergraduate education [4]. DATs are teams of faculty, students, and staff created by our project to address an issue related to undergraduate education in their department. DAT participants work toward enacting sustainable changes by creating new departmental structures and shifting departmental culture. External facilitators from our project team support DAT members in enacting successful changes and in developing capacity as change agents. The DAT model includes multiple components, including a set of Core Principles that guides its design and iterative improvement, common activities and tools for enacting change, and discursive practices and group structures that support functional collaboration.

Here, we present the Core Principles of the DAT model. These principles define the "target" culture that we aspire to foster in our DATs and their departments. Thus, they also undergird the choices we make in designing (and redesigning) the DAT model. We focus on principles for two reasons. First, summarizing a DAT's structure and activities without also externalizing the goals that drive its design would not fully capture what a DAT is. Second, principles are sufficiently abstracted away from the details of the DAT model that they can

\footnotetext{
* The first two authors contributed equally to writing this paper. All other authors are listed alphabetically by last name.
}

be adapted to guide other change efforts in higher education.

Below, we discuss the value of externalizing the principles that guide an intervention's design, present detailed descriptions of the DAT project's Core Principles, and discuss some of their implications for the DAT project and beyond.

\section{DAT MODEL CORE PRINCIPLES}

Principles are generalized guidelines for the design of a learning environment or intervention that bridge theories of learning and practice [5]. They are flexible enough to be adapted to multiple contexts, but are also empirically grounded in prior studies $[6,7]$. Consider our first principle: "Students are partners." The motivation for adopting it might be that student partnerships are known to foster a sense of belonging for students in their departments that will help them succeed in their majors [8]. A department that has implemented this principle may have students who serve on departmental committees, co-teach courses with faculty, or regularly provide their input in other ways. Thus, the implementation of a principle depends on the constraints, norms, and values of a department, even though the motivation for doing so may not. Because of this flexibility and applicability, externalizing an intervention's principles has been a common practice in STEM education (e.g., [9-11]).

A focus on principles is also sensible because change in complex organizations (e.g., university departments) requires attention to multiple layers of culture [2, 3]. We draw from the organizational change literature in defining culture to include both visible "surface" features of an organization (e.g., explicit structures and processes) as well as invisible "deeper" features (e.g., values, norms, beliefs, and assumptions) [3]. These deeper features explain why the surface features exist as they do; the organization's members may know them either consciously or subconsciously.

This multilayered perspective on culture motivates us to connect the visible features of our interventions to our own "invisible" values, articulated through our six Core Princi- 
ples: (1) Students are partners; (2) Work focuses on achieving collective positive outcomes; (3) Data collection, analysis, and interpretation inform decision-making; (4) Collaboration between group members is fun, productive, and rewarding; (5) Continuous improvement is an upheld practice; and (6) Work is guided by attention to diversity and inclusion. We developed these principles by synthesizing literature on organizational change, facilitation, higher education, STEM education, and design thinking. We argue that each principle is valuable and contributes to positive departmental culture; therefore, our design of the DAT model embodies these principles, and our long-term goal is for the principles to spread beyond the DATs and become infused into departmental cultures. We also argue that these principles should inform change efforts beyond ours.

\section{A. Students are partners}

Our concept of "students are partners" includes several key components, including that students have a role in decisionmaking; students and non-students (i.e., faculty and staff) meaningfully share power; students are recognized as having unique expertise and as being an evolving, multifaceted population; and students see themselves as partners. While we recognize that this is a high standard to meet, achieving it can significantly benefit change initiatives. Students bring unique expertise to partnerships, including an understanding of their own cultural backgrounds, experiences, and histories. Thus, a collection of students that represents the breadth of student experiences is best situated to understand how a change will impact diverse student populations [12] and how to help the change succeed, especially when educators come from different backgrounds than the students [13]. Student-faculty partnerships not only benefit the change work itself, but can also increase students' motivation, confidence, sense of belonging and identity within a discipline [8], which can in turn lead to improved academic outcomes.

However, "students are partners" can be challenging to implement. Institutional structures (e.g., faculty committees) are often set up in ways that exclude student participation as full partners. Additionally, for faculty to share power with students challenges the cultural norm of faculty-as-deciders and the belief that it is "natural" for faculty to have more power than students (rather than different power). Therefore, hard work and extra care is necessary to build partnerships that are safe and productive for the students $[8,14]$ and that are true partnerships rather than a façade.

We support this principle by setting the expectation that DATs include student members. Additionally, DAT facilitators work to legitimize student perspectives by affirming students' ideas, ensuring equitable work distributions and inclusive decision-making, and addressing dynamics between student and non-student DAT members that they see as misaligned with this principle, either in the moment during a meeting or via a follow-up in between meetings.

\section{B. Work focuses on achieving collective positive outcomes}

This principle foregrounds having an "outcome-focus" as opposed to a "problem-focus," that is, a focus on what a group is trying to create, rather than what it is trying to eliminate. In problem-focused conversations, individuals often default to their individual preferred solutions, which can result in entrenched disagreement. In contrast, an outcome-focus promotes flexibility and adaptability because any given outcome can be achieved in many ways $[15,16]$. In the context of a change effort, an outcome-focus is grounded by a shared vision, which is a compelling understanding of what a group is trying to achieve that is co-created by members of the group [17]. Such a shared vision provides a group with a strong pull toward a desired outcome, motivating group members. It also binds the group together, encouraging its members to see each other as on the "same team" and to overcome their differences in the service of achieving the vision. Finally, a shared vision helps the group craft short-term goals and define assessment criteria.

However, focusing on outcomes and developing a shared vision can be challenging. Groups often jump to "doing the work" before having a clear understanding of their vision and desired outcomes. Without that shared understanding, the group can easily devolve into conflict about the nature of "the work" because they lack metrics to resolve the disagreement.

One way that we designed toward this principle was through the Ideal Student Activity (inspired by [18]), in which DAT facilitators invite participants to imagine an "ideal student" graduating from their department. DAT members brainstorm characteristics of such a student, such as their knowledge, skills, behaviors, and values. Participants then sort their ideas and note emergent themes. Consensus themes form the backbone of a vision for the DAT's work; because it is collectively generated, this vision helps the DAT members see that they all want to achieve the same kind of outcomes for students in their department.

\section{Data collection, analysis, and interpretation inform decision-making}

This principle involves collecting evidence (e.g., student performance measures, retention data, and background literature) on an ongoing basis. The systematic collection and use of evidence supports better decision-making by allowing for a deeper understanding of the issue at hand and a mechanism for evaluating progress [19]. However, collecting evidence is not enough to ensure good decision-making; careful analysis is also critical. A group that is "analysis-driven" rather than "data-driven" is careful to use analyses and develop interpretations that are appropriate for the given data, and they don't choose questions to answer merely because of the availability of certain data [20]. They also actively identify and avoid bias in interpreting data (e.g., distinguishing observation from inference, developing multiple interpretations of the 
same data, and considering both systemic and individualistic explanations).

Two common failure modes with respect to this principle are making decisions based on meaningless evidence or gathering an abundance of good evidence without a plan for how to use it meaningfully. In either case, decision-making will be highly susceptible to bias through, e.g., over-reliance on personal anecdote [21] or confirmation bias, in which individuals interpret data in ways that fit their existing ideas [22].

DAT facilitators support this principle by implementing structured activities for working with evidence. For example, facilitators will ask DAT members to predict what a data set will show before analyzing it. After the analysis, they will discuss how their predictions aligned with reality. This activity helps DAT members develop reflective analytic skills, avoid confirmation bias, and confront their assumptions.

\section{Collaboration between group members is fun, productive, and rewarding}

This principle focuses on authentic collaboration, in which all group members can participate in group activities and contribute to decision-making. For this to happen, the group must have productive group norms, the group's (often implicit) patterns of behaviors and interactions. Productive group norms support constructive disagreement, consideration of multiple viewpoints, and equitable ways of doing work [20]. Establishing and maintaining these norms helps teams work effectively. Authentic collaboration also supports members in feeling like part of a community, which is defined, in part, by its shared values, coordination by its members to achieve collective outcomes, and common activities that bind the group together [23]. Together, these features build trust and mutual dependence among group members, which leads to more productive work. Moreover, people are more likely to engage in work when doing so meets their emotional needs (e.g., connection to others, enjoyment, and feeling valued) [15].

This principle can be challenging because it violates expectations about how academics "should" interact with each other (e.g., focusing on "work" without the "distraction" of personal discussions). Additionally, talking about "process" is both unfamiliar and uncomfortable for many people, so being explicit about norms does not come naturally to groups.

Within DATs, we enact eight Norms of Collaboration, specific behaviors that support groups in working well together (e.g., "paraphrase others' ideas") [20]. Facilitators introduce these norms in meetings, discuss their utility, and intentionally model them. DAT participants practice using the norms in meetings and indicate that they value their explicit use.

\section{E. Continuous improvement is an upheld practice}

This principle centers on the idea that "improvement" needs to be valued by all members of a group on an ongo- ing basis. Educational problems rarely "stay solved" on their own; instead, they need continuous attention to prevent backsliding $[19,24]$. A valuable way to support continuous improvement is to design a change process that is iterative. Iterative processes lead to better outcomes because they support idea generation and continual assessment of progress [25]. Continuous improvement also requires attention to maintaining momentum, both internally and from external supporters. The ongoing articulation of incremental progress toward their vision can help a group maintain both forms of momentum. Taken together, a group that aligns its change effort with all of these components of continuous improvement will be more likely to achieve sustainable change.

A challenge to engaging in continuous improvement is the belief that change is an "event" rather than a process (i.e., that once a change has been made, sustaining it doesn't require more work). This belief is rarely true for complex change efforts and can often lead to the investment of time and energy into a change that is unlikely to last in the long term.

DAT facilitators help DAT members distill short-term achievable goals from their long-term visions. Early implementation and assessment of those goals help participants develop deeper understanding of their focal issue and identify roadblocks. These "early wins" also support internal momentum and demonstrate success to other stakeholders.

\section{F. Work is guided by attention to diversity and inclusion}

This principle involves recognizing and mitigating systemic oppressive power imbalances. Racism, sexism, and other forms of marginalization exist within university departments [26]. Without explicit attention to these forms of marginalization, groups can unintentionally reproduce problematic behaviors. Therefore, group members must recognize that they are all personally responsible for working against oppressive structures in their organizations and considering the impacts of their decisions on marginalized groups. A starting point for aligning with this principle is for groups to recruit a diverse membership across many relevant dimensions of marginalization. This principle supports groups in recognizing that issues of oppression are inextricably linked to their work and must be proactively addressed if the group does not want to reproduce harmful patterns themselves.

This is perhaps the most difficult principle to achieve in practice. In general, dominant American culture denies the existence of systemic oppression and withholds tools for engaging with it productively [27]. Thus, it is challenging, emotionally charged work for people from dominant populations to recognize oppression, its systemic nature, and the role they play in maintaining it. Moreover, it is easier to falsely believe that one's work is anti-oppressive than to challenge oneself to higher standards that align with this principle.

To support this principle, we set the expectation that DATs will have diverse memberships (e.g., with respect to department role, gender, ethnicity, and country of origin). This al- 
lows them both to develop better solutions [28] and to identify how changes will impact a diversity of populations. DAT facilitators attend to how marginalization can play out in meetings and work toward ensuring that all voices are valued. Facilitators also discuss characteristics of organizations that lead them to be oppressive and "antidotes" of these characteristics that DATs can implement in their work [29].

\section{DISCUSSION AND IMPLICATIONS}

We have summarized the Core Principles that our project team uses to guide the development of the DAT model, inform facilitation, and define the culture towards which we try to move DATs (and their departments). While we discussed these principles independently, they are actually mutually reinforcing. For example, the iteration implied by continuous improvement requires a set of outcomes that emerge from a shared vision; the process of developing a shared vision builds community; and attention to inclusion involves seeing all department members, including students, as potential partners. Additionally, while there are many ways in which DATs have embodied these principles in practice, we have also encountered challenges in their practical implementation. In future work, we will articulate the mutually reinforcing connec- tions among the principles further, and we will discuss the details of our implementation and self-critically reflect on how we can better support DATs in meeting those principles.

Beyond DATs, we believe that these principles are widely applicable to the design of other efforts trying to support improved undergraduate education or departmental change (e.g., Faculty Learning Communities, departmental committees, instructional design support). These principles represent our synthesis of literature from higher education, STEM education, design thinking, facilitation, and organizational change. We find that these sets of literature speak to each other in generative ways, and we therefore see this synthesis as valuable for broadening the PER and DBER communities' conceptualizations of what their change efforts could aspire to achieve. By briefly presenting some ways in which we have connected theory with practice, we hope to encourage others to either build on these principles or develop their own to help further develop theory-driven models for enacting change in academic departments.

\section{ACKNOWLEDGMENTS}

We thank NSF for funding this work under DUE-1626565 and Dimitri Dounas-Frazer for his helpful feedback.
[1] C. Henderson, A. Beach, and N. Finkelstein, J. Res. Sci. Teach. 48, 952 (2011).

[2] A. Kezar, How Colleges Change: Understanding, Leading, and Enacting Change (Routledge, 2014).

[3] E. H. Schein, Organizational Culture and Leadership (JosseyBass, 2010).

[4] D. L. Reinholz, J. C. Corbo, M. H. Dancy, and N. Finkelstein, Learn. Communities J. 9 (2017).

[5] V. Svihla and R. Reeve, Design as Scholarship: Case Studies from the Learning Sciences (Routledge, 2016).

[6] B. Kirshner and J. L. Polman, Yearb. Natl. Soc. Study Educ. 112, 215 (2013).

[7] M. C. Linn, P. Bell, and E. A. Davis, in Internet Environments for Science Education, edited by M. C. Lin, E. A. Davis, and P. Bell (Lawrence Erlbaum Associates Publishers, 2004), pp. 315-339.

[8] L. Mercer-Mapstone, S. L. Dvorakova, K. Matthews, S. Abbot, B. Cheng, P. Felten, K. Knorr, E. Marquis, R. Shammas, and K. Swaim, Int. J. Stud. Partn. 1 (2017).

[9] R. A. Engle and F. R. Conant, Cogn. Instr. 20, 399 (2002).

[10] A. S. Jurow, R. Hall, and J. Y. Ma, J. Learn. Sci. 17, 338 (2008).

[11] K. E. Metz, Cogn. Instr. 22, 219 (2004).

[12] K. Tobin, Teach. Educ. 17, 133 (2006).

[13] M. Sabella, A. V. Duzor, and F. Davenport, in Physics Education Research Conference 2016, edited by D. L. Jones, L. Ding, and A. L. Traxler (2016), pp. 288-291.

[14] K. E. Matthews, Int. J. Stud. Partn. 1 (2017).

[15] D. L. Cooperrider, D. Whitney, and J. M. Stavros, The Appreciative Inquiry Handbook: For Leaders of Change (BerrettKoehler, 2008).
[16] S. Elrod and A. Kezar, Tech. Rep., AACU (2015).

[17] P. M. Senge, The Fifth Discipline: The Art and Practice of the Learning Organization (Doubleday Currency, 1990).

[18] M. Savina, D. Gross, and C. Danielson, Ideal student exercise, https://serc.carleton.edu/NAGTWorkshops/departments/ degree_programs/idealstudent.html, accessed July 2, 2018.

[19] The Coalition for Reform of Undergraduate STEM Education, Tech. Rep., AACU (2014).

[20] R. J. Garmston and B. M. Wellman, The Adaptive School: A Sourcebook for Developing Collaborative Groups (Christopher-Gordon Publishers, 2009).

[21] D. Kahneman, Thinking, Fast and Slow (Farrar, Straus and Giroux, 2013).

[22] R. S. Nickerson, Rev. Gen. Psychol. 2, 175 (1998).

[23] E. Wenger, Communities of Practice: Learning, Meaning, and Identity (Cambridge University Press, 1999).

[24] A. S. Bryk, L. Gomez, and A. Grunow, in Frontiers in Sociology of Education, edited by M. T. Hallinan (Springer, 2011), pp. 127-162.

[25] T. Brown, Harv. Bus. Rev. 86, 84 (2008).

[26] E. Seymour and N. M. Hewitt, Talking about Leaving: Why Undergraduates Leave the Sciences (Westview Press, 1997).

[27] K. K. Kumashiro, Rev. Educ. Res. 70, 25 (2000).

[28] F. J. Milliken, C. A. Bartel, and T. R. Kurtzberg, in Group Creativity: Innovation through Collaboration, edited by P. B. Paulus and B. A. Nijstad (Oxford University Press, 2003), pp. 32-62.

[29] T. Okun, White supremacy culture, https://collectiveliberation. org/wp-content/uploads/2013/01/White_Supremacy_Culture_ Okun.pdf, accessed June 27, 2018. 